\title{
Gas Exchange and Exhaust Condition Modeling of a Diesel Engine using the Engine Dynamics Library
}

\author{
Johan Dahl $\dagger$ Daniel Andersson $\ddagger$ \\ $\nmid$ Volvo Group Truck Technology, Control Systems, Gothenburg, Sweden \\ †Modelon AB, Lund, Sweden
}

\begin{abstract}
In this paper the newly developed Engine Dynamics Library is presented. Ever increasing consumer and regulatory demand for improved fuel economy and lower emissions forces the engines and Engine AfterTreatment Systems (EATS) to be improved continuously. Since the complete system is very complex, models are useful in cost effectively developing new control strategies and select hardware. The library is based on a mean-value combustion model and the focus lies on modeling the gas exchange with real-time like simulation times, useful for engine optimization and for evaluation of control strategies. The library contains models of the standard engine components such as manifolds, pipe, turbines, compressors, valves, mechanics, etc. Simulation results from Dymola for a $13 \mathrm{~L}$ Volvo truck engine demonstrate that the model captures the transient flow and temperatures and emission trends, and has sufficient accuracy to be useful in engine optimization. The physical modeling approach allows for virtual prototyping by replacing individual components, which is an important advantage over black-box modeling. It is shown that the model captures essential system properties in the gas exchange, such as non-minimum phase behavior and sign reversal for VGT and EGR valve actuation. The model has been calibrated using surface fitting of maps and leastsquares estimation of parameters in Matlab, as well as parameter optimization using JModelica and FMI.
\end{abstract}

Keywords: Engine modeling; Engine simulation; Air Gas management

\section{Introduction}

As the requirements on the engine and EATS become more strict, a new development process of control strategies and hardware concept selection is needed as only using engine test cells and vehicles in the development process is too time consuming and expensive.
In the new development process at Volvo, SoftwareIn-the-Loop (SIL) simulations are used more extensively in the control strategy and hardware development. With the introduction of US10 and soon EU6 legislation ultra low on-road emissions are required. Future emission legislation will also include $\mathrm{CO}_{2}, \mathrm{~N}_{2} \mathrm{O}$ and $\mathrm{NO}_{2}$ [1]. To fulfill these requirements with optimal fuel consumption, the significant interaction between the engine and EATS must be considered and control strategies for both components need to be optimized together [2]. This requires good engine models with accurate modeling of the engine out conditions. In particular, focus has been on predicting the sensitivity of the dynamic response and engine exhaust temperature with respect to the air gas management. Issues about control system design or strategy are not in the scope of this paper. Nevertheless, a good physical model of the engine provides useful insights for both the control system designers and hardware selection. The engine model is also useful for finding suitable requirements of the EATS system. For example the emission transient response can be a limiting requirement for the needed volumes of the Diesel Oxidation Catalyst (DOC), Diesel Particulate Filter (DPF) and Selective Reduction Catalyst (SCR) in order to fulfill the EU6 emission legislation.

In this paper the Engine Dynamics Library (EDL) is presented. The library is implemented in Modelica and consists of mean-value models of standard engine components. The focus of the model has been on capturing the transient engine response and the engine outlet conditions as these features are important for the total engine and EATS optimization. Comparison results between test cell measurements and simulation results of a 13 liter Volvo truck engine certified for the Post New Long-Term (PNLT) emission legislation, introduced in 2009, are presented. The Engine Dynamics Library is a new commercial library offered by Modelon. 


\section{Engine modeling in Dymola}

Today several tools exist in which physical or semiphysical models can be implemented. Dymola [3], which is based on the open standard Modelica language, was chosen as the tool for developing an engine model library. The main reasons for choosing Modelica are the flexibility, expressiveness and openness of the language, as compared to domain specific tools, and the possibility to extend tools and libraries with in-house IP and know-how. Others have demonstrated that Modelica is suitable for engine modeling $[4,5]$, but the focus has not been on gas exchange modeling or predicting the exhaust gas temperature entering the EATS.

The following sections describe EDL and the component and medium models that have been implemented.

\subsection{Library structure}
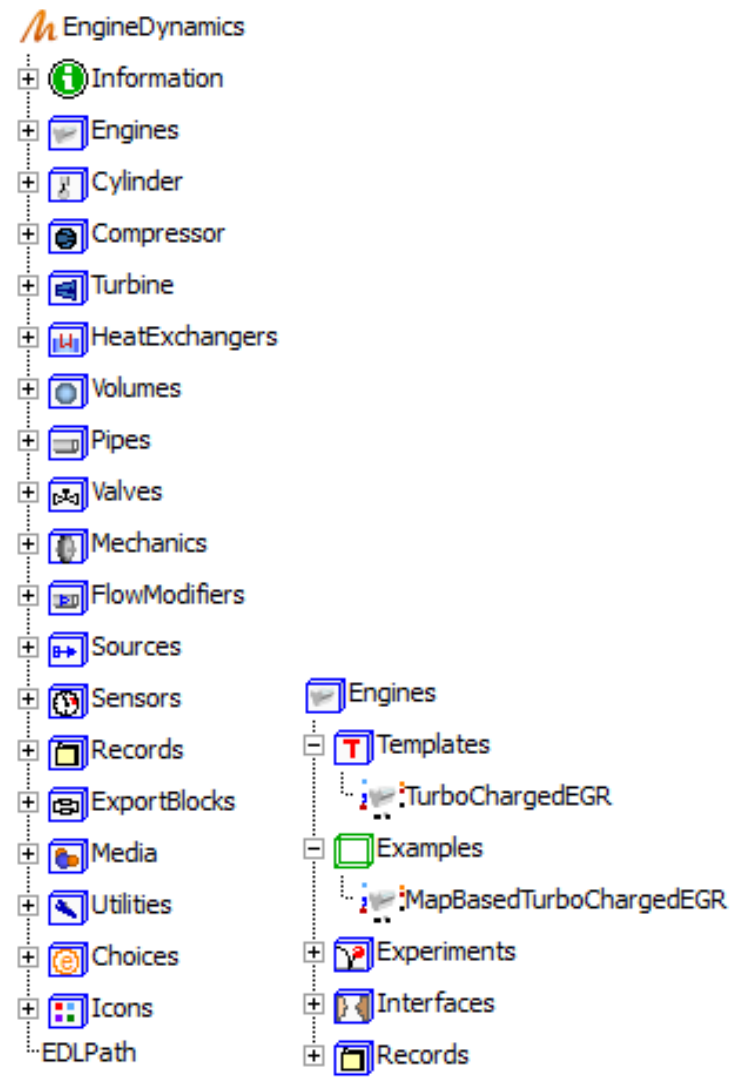

Figure 1: EDL and sub packages (left), Engines package (right)

The structure of EDL is shown to the left in Fig. 1. The library is divided into packages for each physical component, plus some additional packages for supporting components and classes. There is also a package named Engines, shown to the right of Fig. 1, which contains examples of configured engine models and experiments.

EDL is not based on the Modelica.Media or Modelica.Fluid packages. Medium property models and base classes for fluid systems modeling are based on classes in the Modelon Base Library, which is delivered with EDL. EDL share base classes with Modelon's Liquid Cooling Library (LCL), Heat Exchanger Library (HXL) and Vehicle Dynamics Library (VDL), making them all compatible. The libraries can be used together for different kinds of vehicle analysis, for example EDL, LCL and HXL together forms a powerful solution for thermal management analysis, and EDL and VDL can be used together for drivability analysis.

\subsection{Cylinder}

The cylinder component (Fig. 2) is based on a mean value combustion model as described in [6]. The component boundary conditions are boost pressure and temperature, exhaust manifold pressure, 0000006 Figure 2: Cylinder engine speed, fuel injection and other control signals. The empirical correlations described in the following sections (often 2-dimensional maps) can easily be replaced by any equation based models, for example simple qualitative models found in literature, regression models or neural network models.

\section{Flow model}

The cylinder mass flow is modelled by means of a volumetric efficiency defined as:

$$
\dot{m}_{\text {charge }}=\rho_{\text {in }} \cdot \lambda\left(p_{\text {Boost }}, \omega_{e}\right) \cdot \frac{V_{d}}{N} \cdot \frac{\omega_{e}}{2 \pi}
$$

where $\lambda$ is the volumetric efficiency, $V_{d}$ is the displaced volume, $N$ is the number of revolutions per cycle, $p_{\text {Boost }}$ is the inlet manifold pressure and $\omega_{e}$ is the engine rotational speed. $\lambda\left(p_{\text {Boost }}, \omega_{e}\right)$ is modelled by a two-dimensional map obtained from measurements.

\section{Torque model}

For the torque model we define brake mean effective pressure, $p_{m e}$ and fuel mean effective pressure, $p_{m \varphi}$, as:

$$
p_{m e}=\frac{T_{e} \cdot 4 \pi}{V_{d}} \quad p_{m \varphi}=\frac{H_{l} \cdot m_{\varphi}}{V_{d}}
$$

where $T_{e}$ is the engine torque, $H_{l}$ is the fuel lower , heating value and $m_{\varphi}$ is the mass of fuel burnt per 
combustion cycle. The engine efficiency can then be written as:

$$
\eta_{e}=p_{m e} / p_{m \varphi}
$$

Following the Willans Approximation [6], a torque model on the following form is implemented:

$$
p_{m e}=e\left(p_{m \varphi}, \omega_{e}\right) \cdot p_{m \varphi}-p_{m e 0 f}\left(\omega_{e}\right)-p_{m e 0 g}
$$

where the energy conversion efficiency, $e$ is modelled by a two-dimensional map obtained from measurements, the mechanical friction, $p_{m e 0 f}$, is mapped from engine speed, $p_{m e 0 g}$, is the cycleaveraged pressure difference between inlet and exhaust manifolds.

\section{Exhaust gas properties}

The outlet exhaust gas temperature is mapped from engine speed and injected fuel. The transferred heat to the cylinder block is obtained from energy balance over the component boundaries.

The composition of species in the exhaust gas is modelled by a stoichiometry matrix for the combustion. Complete combustion of the injected fuel is assumed. The $N O_{x}$ and soot generation is modelled by a regression model [7] on the form:

$$
y(t)=\phi^{T} \theta+e(t)
$$

where $y=\left(C_{N O x}, C_{\text {Soot }}\right)^{T}$ are the $N O_{x}$ and soot concentrations of the exhaust gas, the regressor $\phi=$ $\left(1, u_{1}, u_{1}^{2}, \ldots, u_{1}^{N}, u_{2}, \ldots\right)^{T}$ contains the first and higher order terms of the following signals:

- Injected fuel amount, $m_{f}$

- Fuel injection timing, $\zeta$

- Needle opening angle (controls the fuel injection pressure), $\beta_{f}$

- $\mathrm{CO}_{2}$ concentration in the inlet manifold, $\mathrm{C}_{\mathrm{CO} 2}$

- Inverse stoichiometric air to fuel ratio, $\lambda^{-1}$

- Engine speed, $\omega_{e}$

$\theta$ are the model parameters and $e$ is the model error. In the experiment described in section 3.2, all of the input signals to the model come directly from model control signals or boundary conditions, except for the inlet manifold $\mathrm{CO}_{2}$ concentration and air to fuel ratio. These variables are simulated in the engine system model and the simulated values are used as inputs to the emission model.

\subsection{Compressor and turbine}

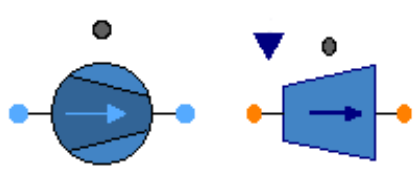

Figure 3: Compressor and VGT

The compressor and variable geometry turbine (VGT) components (Fig. 3) are both parameterized by maps for mass flow rate and isentropic efficiency. The components model a polytropic thermodynamic process with mechanical power crossing the component boundary via a rotational mechanical flange. Quasistatic balance equations for conservation of substance mass and energy are used, i.e. storage of mass and energy is not considered and the outlet properties respond instantly to property changes of the inlet flow. These equations assume:

- The amount of mass inside the component is small compared to that in the upstream and downstream pipes, which is covered by volume components connected to these components.

- The heat capacity of the solid parts are lumped together with the wall heat capacities of the volume components connected upstream and downstream of these components.

- The rotational kinetic energy of the solid parts is modeled by a separate inertia component connected to the rotational flange connector of these components.

The mapped isentropic efficiency, $\eta_{i s}$, defines the deviation from an isentropic process [8].

$$
\begin{array}{rlr}
\eta_{\text {is }}=\frac{h_{\text {out }, \text { isentropic }}-h_{\text {in }}}{h_{\text {out }}-h_{\text {in }}} & \text { (Compressor) } \\
\eta_{\text {is }}=\frac{h_{\text {out }}-h_{\text {in }}}{h_{\text {out }, \text { isentropic }}-h_{\text {in }}} & \text { (Turbine) }
\end{array}
$$

where $h_{\text {in }}$ is the inlet specific enthalpy, $h_{\text {out }}$ is the outlet specific enthalpy and $h_{\text {out }}$,isentropic is the outlet specific enthalpy of an isentropic process.

The variable geometry turbine is modeled using several maps of isentropic efficiency and mass flow rate for different positions, the properties are interpolated linearly between the mapped geometry settings. The turbine model currently contains no compensation for the upstream pressure oscillations. Internal losses 
from heat transfer to the housing and mechanical friction are currently modeled as a constant efficiency factor. The turbo moment of inertia is captured by a separate inertia component connected between the compressor and turbine components in the engine system model.

\subsection{Heat exchangers}

A quasi-static heat exchanger model with table based efficiency is implemented in EDL. It does not contain storage of mass or energy and the outlet fluid properties respond instantly to inlet property changes. The component has interchangeable friction models with different

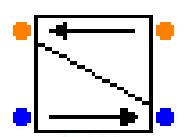

Figure 4: Heat exchanger levels of detail for the primary and secondary flow channels. A model on the following form was chosen because it is easily calibrated to fit measured data:

$$
d p=f \cdot \frac{\rho}{2} \cdot v^{n}
$$

Here $d p$ is the pressure drop over the channel, $f$ is the friction factor, $\rho$ is the fluid density, $v$ is the flow velocity and $n$ is a constant. Note that for $n=2$, this corresponds to the Darcy-Weisbach equation for pressure loss due to friction in a pipe. The constants $f$ and $n$ are chosen to fit measurement data.

The heat transfer is modeled by defining heat exchanger efficiency as $\varepsilon=Q / Q_{\max }$. The maximum transferable heat $Q_{\max }$ is calculated from the heat capacity flow and inlet temperatures of the two channels. The model is parameterized by specifying a twodimensional map for the efficiency from the mass flow rates in the two channels.

\subsection{Volumes}

All fluid mass and energy storage is modelled in volume components by dynamic mass and energy balance equations. An ideal mixture is assumed and a number of different components are available,

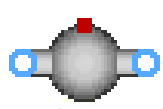

Figure 5: Two port air volume which have different port configurations. The volume models have the option to consider wall heat capacity, heat transfer between fluid and wall (constant heat transfer coefficient model) and heat transfer to the surroundings. There is a special volume model for the inlet manifold that can handle incoming flow in a different medium model representation by mapping the fluid species between the mass fraction vectors of the two medium models. This is necessary if separate models for air and exhaust gas are used. Outgoing flows from the volume carry the average medium properties of the total volume.

\subsection{Pipes}

The pipe models provided in the library consider pressure drop due to friction and optionally also heat transfer effects. Several friction models can be chosen, but also here eq. 8 is used. The heat transfer model is interchangeable as

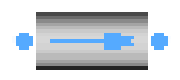

Figure 6: Air pipe model well, with the options: 1) Constant heat

transfer coefficient, 2) Dittus-Boelter correlation for forced convection in turbulent flow (Coefficients can be adjusted by the user). Optionally a dynamic momentum balance can be used.

\subsection{Valves}

There are a number of valve models available in EDL. The first one is designed to be easily parameterized from measured data. It defines a flow equation for the fully opened setting as eq. 8 . The valve characteristics are represented by means of a relative open area that is

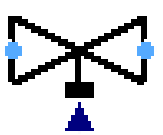

Figure 7: Valve model governed by the actuation signal. Linear, quadratic and tabulated characteristics are available. The second one is implemented according to the IEC 534/ISA S.75 standards for valve sizing. It accounts for fluid compressibility effects, as well as choked conditions. For the engine model presented in this paper, the first model is used because it is easier to parameterize from measurements and choked conditions do not occur under normal operation.

A butterfly type valve model has been implemented as well, including flap mechanism, torque generation on the flap by the gas flow and mechanical friction.

\subsection{Medium models}

The medium property models are implemented as replaceable packages with high flexibility, similar to that of the Modelica.Media package. Ideal gas mixtures based on the NASA coefficients [9] can be created and used.

In addition to this, a simplified medium model assuming a linear function for specific heat capacity of temperature, $C_{p}(T)$, has been implemented for performance reasons. By definition, the specific enthalpy function, $h(T)$, will become quadratic in temperature 
under this assumption. In static component models, the upstream temperature $T(h)$ is calculated from the specific enthalpy of the inlet fluid connector. An explicit function for this calculation greatly improves simulation performance for system models with several such components, as the non-linear systems of equations can be reduced or completely avoided. The medium models are compatible, so all component models can carry any of the medium model types.

Available in EDL are some pre-defined mixtures, used as air or exhaust gas models. The components included are $\mathrm{CO}_{2}, \mathrm{H}_{2} \mathrm{O}, \mathrm{O}_{2}, \mathrm{~N}_{2}$ and $\mathrm{Ar}$ for both NASA and linear $C_{p}(T)$ models. Also a single component dry air model is provided. To model emissions, some pre-defined exhaust gas mixtures include trace components for $N O_{x}$, Soot, $H C$ and $C O$. The trace components are assumed to be carried by fluid flow but don't affect the thermodynamic properties of the fluid.

\subsection{Mechanical}

Basic rotational mechanical components are available in EDL, such as inertia and ideal gear models. The mechanical connectors of the turbo components and cylinder component are compatible with the mechanical components in the Modelica Standard Library.

\section{Engine system model}

A 13 liter Volvo engine certified for the Post New Long-Term (PNLT) emission legislation has been modeled using EDL. The engine is equipped with variable geometry turbine, exhaust gas recirculation governed by a valve, throttle, EGR cooler, intercooler and unit injectors. The purpose of the simulation model is to perform similar experiments that are performed in engine test cells, where the engine is mounted to an electrical dynamometer which directly controls the engine speed.

\subsection{Model description}

The engine system model is configured as shown in Fig. 8. The upper left connector is the air inlet connector that should be connected externally to a component defining air temperature and pressure boundary conditions. The components in the air path are connected to represent the engine system design, indicated with light blue in the figure. First there is a pipe component modeling the pressure drop over the air filter (1). Then follows compressor (2), intercooler (3) and throttle (4)

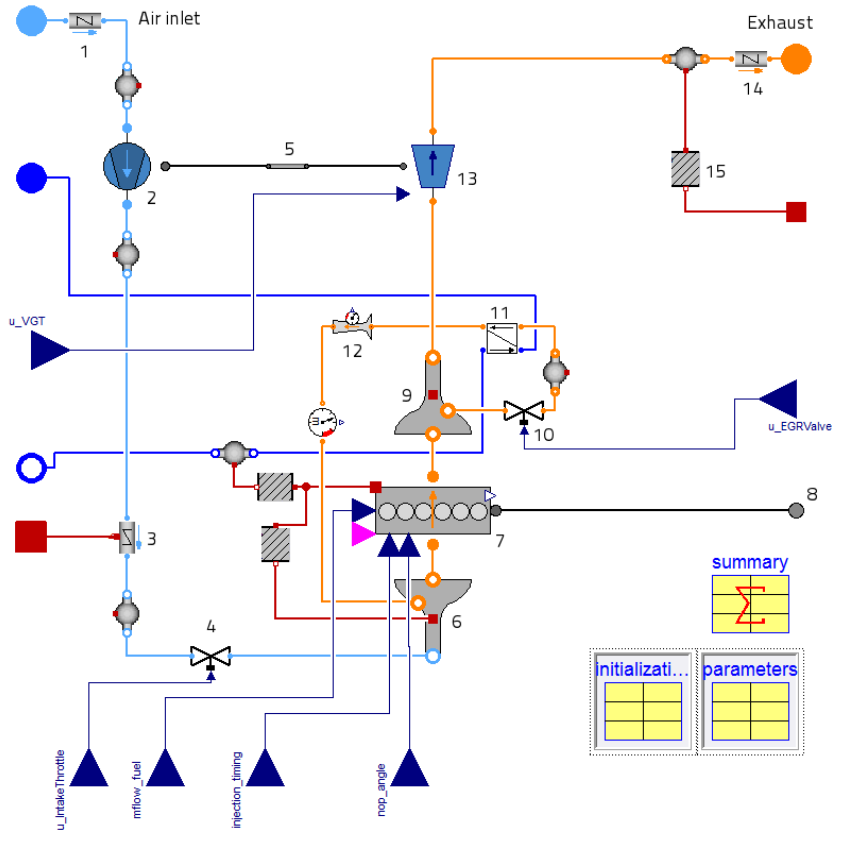

Figure 8: Engine system model with: Air filter (1), Compressor (2), Intercooler (3), Throttle (4), Turbo inertia (5), Inlet manifold (6), Cylinder block (7), Drive shaft (8), Exhaust manifold (9), EGR valve (10), EGR cooler (11), Venturi (12), VGT (13), Muffler (14), Heat transfer (15, and more)

components, each separated by volume components. The compressor is connected to an inertia model (5) that is also connected to the VGT component (13).

The throttle in the lower left is connected to the inlet manifold component (6). This is a volume model that also accounts for the thermal mass of the wall and heat transfer between the gas and wall. The inlet manifold has two more connectors for gas (orange). One is connected to the cylinder block and the other is the inlet for EGR gas.

The cylinder block (7) has a rotational connector for the drive shaft that is connected to an external connector to the right in the figure (8). It is also possible to enable a support connector for the reactive torque, but it is not used here. There are real input signal connectors for injected fuel, injection timing and needle opening angle. The exhaust gas port is connected to the exhaust manifold (9), which is also a volume model including thermal mass of the wall. There is an outlet port for the exhaust gas recirculation path that is connected directly the the EGR valve (10). This is connected to a volume and then to the EGR cooler (11) and venturi (12). The venturi component is a pure sensor model that does not affect the gas flow rate or properties. The EGR gas path is then fed back to the inlet manifold. 
For each volume model there is a unique pressure and temperature state introduced. As a consequence of the model layout the flow through the EGR valve is calculated from the pressure difference between the exhaust manifold and EGR volume components. The pressures are calculated during model simulation by means of numerical integration.

The exhaust manifold is also connected to the turbine component (13). Additionally, the turbine has an input signal for varying the geometry, a rotational flange connector and an outlet gas connector. The turbine component calculates a torque that is generated on the flange. Thus, the turbo rotational speed is obtained during simulation by integration of the dynamic momentum equation introduced in the inertia component, with torque terms from the turbine and compressor components. After the turbine the gas is fed to a volume model and then a pipe model that accounts for the pressure drop over the muffler (14).

The volume model in the exhaust path has a thermal connector (red square) that holds the wall temperature of the exhaust pipe. This is connected to a heat transfer component (15) that contains a linear heat transfer equation. This is also connected to an external heat connector where the ambient temperature should be provided as boundary condition. Such heat transfer components are also used to cover heat transfer between the cylinder block and coolant water, and between cylinder block and inlet manifold. The coolant path is indicated with dark blue connections. The set of connector variables in the air, gas and water connectors are identical. Only the color differ for a clearer visual model representation.

\subsection{Simulation model}

The engine model described above can be used in various simulation models or virtual experiments. Simulation models are created by instantiating the engine model and assigning values or signal to boundary conditions and input control signals. The following signals from the engine electrical control unit (EECU) are set as input signals:

- Injected fuel, injector timing and needle opening angle (controls the fuel pressure)

- VGT, EGR valve and throttle positions

The following physical boundary conditions are set:

- Engine coolant temperature and mass flow rate

- Ambient air temperature and pressure

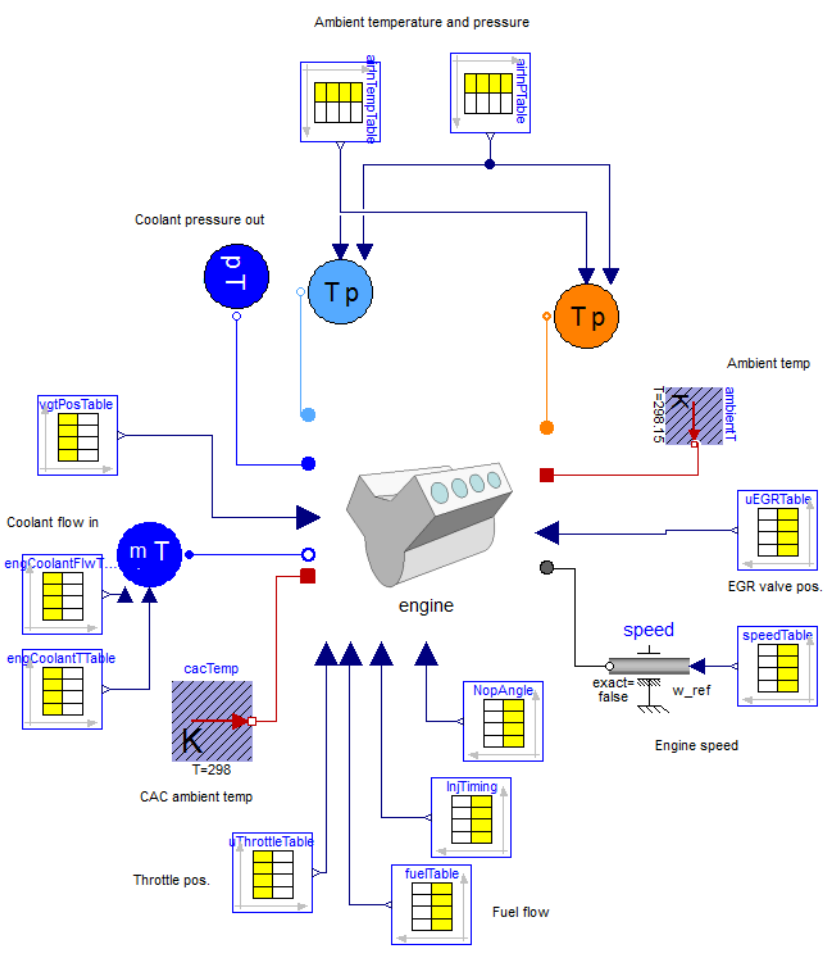

Figure 9: Simulation model of the engine in a test cell. The engine component corresponds to the engine model as shown in Figure 8.

\section{- Engine driveshaft speed}

This experiment is set up in Dymola, as shown in Fig. 9. The centered engine icon represents the engine model as shown in Fig. 8. The components with table icons are used to read signals from the engine test cell measurements from an external file. The engine component need not be connected directly to source components, but could be used in larger system models together with drive line, vehicle dynamics, coolant system or exhaust after treatment system models.

\section{Calibration}

The calibration is done component by component. The benefit with this approach is that it is possible to change a component and only recalibrate the new component without needing to recalibrate the whole systems. Validation is performed both component by component and for the overall system using steadystate and dynamic data. The exhaust gas thermal dynamics is calibrated using an exhaust gas path subsystem model. 


\subsection{Static correlations}

The calibration of the static engine correlations is performed in Matlab using steady state measurement data. Flow model parameters for pipe, valve and heat exchanger models are calibrated with a Least-Squares method using static engine screening data. For the compressor and VGT the maps supplied by the manufacturer were used. Heat exchanger measurements were also supplied separately, and not identified from the screening data. The maps for energy conversion efficiency, volumetric efficiency and exhaust gas temperature used in the cylinder component were calibrated using the surface fitting tool gridfit [10]. The calibration data for this component consisted of a partial load map collected from an engine test cell. For the valves, one dimensional look-up tables for relative open area from the control signal were created. Some results from the calibration procedure are presented in the following figures. Fig. 10 shows the fitted surface for volumetric efficiency together with measurements. Fig. 11 shows the measured mass flow rate through the intercooler at different pressure drops together with a calibrated model using equation 8 . Fig. 12 shows the fitted look-up table for throttle relative open area.

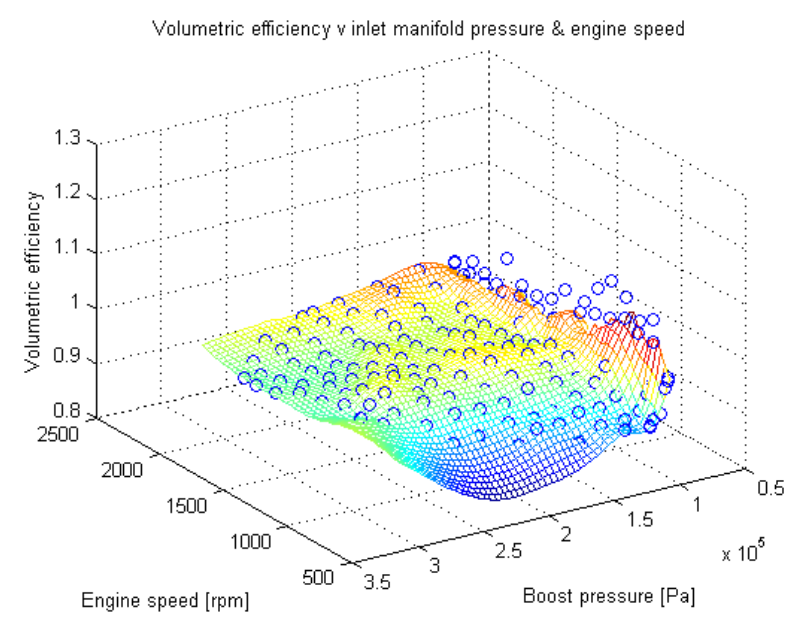

Figure 10: Volumetric efficiency map, fitted map and measured data

\subsection{Emission model}

The linear regression model is calibrated by least squares estimation [7]. For calibration, the initial 10 minutes of the dynamic JE05 cycle, further described in section 5, were used. The remaining 20 minutes are then used for validation of the calibration result. The

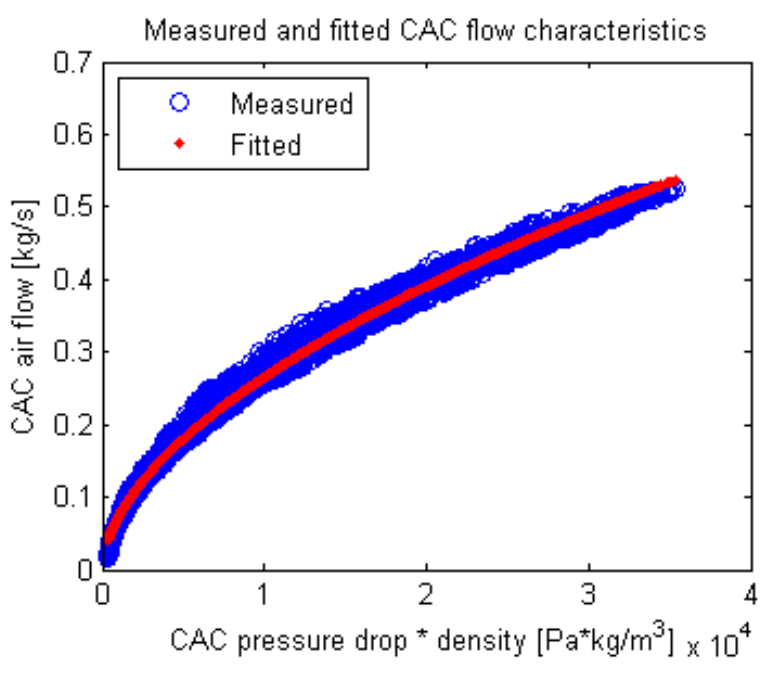

Figure 11: Intercooler flow friction model

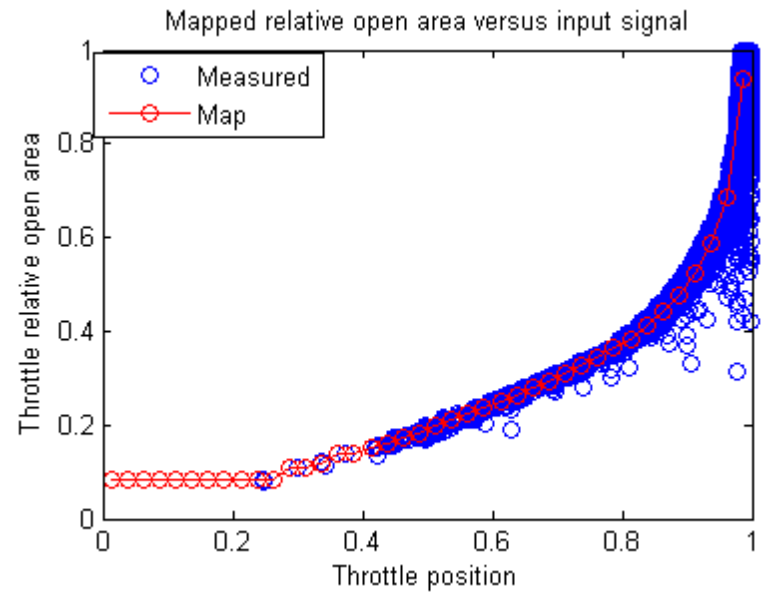

Figure 12: Throttle relative open area

following regressor found to best best result

$$
\begin{aligned}
\phi=(1, & m_{f}, m_{f}^{2}, m_{f}^{3}, \\
& \zeta, \zeta^{2}, \zeta^{3}, \\
& \beta_{f}, \beta_{f}^{2}, \beta_{f}^{3}, \\
& C_{C O 2}, C_{C O 2}^{2}, C_{C O 2}^{3}, \\
& \lambda^{-1}, \lambda^{-2}, \lambda^{-3}, \\
& \left.\omega, \omega^{2}, \omega^{3}\right)
\end{aligned}
$$

\subsection{Parameter optimization in JModelica.org}

JModelica.org [11] has been used for optimization of model parameters for heat transfer and thermal dynamics in the exhaust gas path. The method used is the derivative free Nelder-Mead simplex method $[12,13]$. Derivative free methods do not require that the model provides derivatives of the objective function with respect to tuner variables. That makes them well suited 
for optimization of more complex models, and model modifications for optimization purposes are not necessary. The following parameters were optimized to obtain the best possible result for the exhaust gas temperature during transient cycles:

- Thermal conductance between exhaust gas and wall

- Heat capacity of the exhaust pipe wall

- Thermal conductance between the wall and the surrounding air

The dynamic exhaust gas temperature response, presented in Fig. 23, is very different from the instantaneous outlet gas temperature from the quasi-static VGT model. This is both due to thermal mass of the metal parts, and heat transfer to the surrounding air. The heat capacity and thermal conductances mentioned above model the dynamic exhaust temperature response from the VGT outlet temperature. The initial 10 minutes of the JE05 cycle were used for parameter optimization. The remaining 20 minutes are then used for validation of the calibration result.

\section{Validation}

The models have been validated, both by individual component experiments, and by complete engine system simulation. The used data was collected from an engine test cell and consisted of partial load map data and of the Japanese emission cycle, JE05. The JE05 cycle is one of the legislation requirements in the Post New Long-Term (PNLT) legislation.

\subsection{Turbo model validation}

The turbo model with rotational speed as dynamic state was validated separately with boundary conditions from a partial load map. An experiment model is set up where a compressor and VGT component are connected with an inertia model in between. Upstream and downstream pressure and temperatures and VGT position are prescribed and the resulting mass flow rate, outlet temperature and rotational speed are validated for the compressor and turbine models. Fig. 13 shows a comparison of the turbo flow rates. In Fig. 14 the turbo model outlet temperatures are shown.
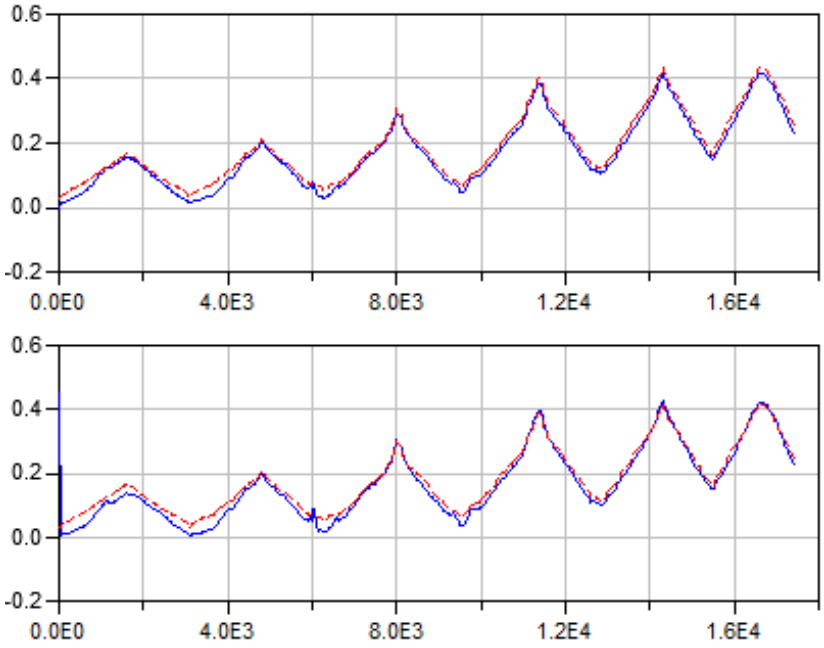

Figure 13: Turbo model validation. Top: Exhaust flow rate $[\mathrm{kg} / \mathrm{s}]$, simulated (solid) and measured (dashed). Bottom: Air flow rate $[\mathrm{kg} / \mathrm{s}]$, simulated (solid) and measured (dashed)

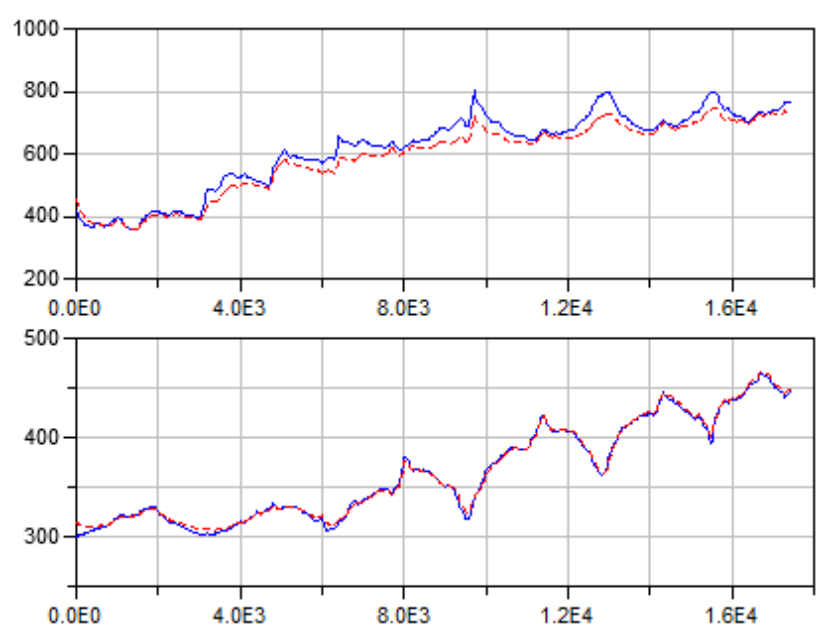

Figure 14: Turbo model validation. Top: Turbine outlet temperature $[\mathrm{K}]$, simulated (solid) and measured (dashed). Bottom: Compressor outlet temperature $[\mathrm{K}]$, simulated (solid) and measured (dashed)

\subsection{EGR model validation}

The EGR valve model is validated with part load map data. Upstream and downstream pressures are prescribed and the simulated EGR flow rate is compared to measurements. The result is presented in Fig. 15.

\subsection{Verification of non-minimum phase and sign reversal}

An engine equipped with VGT and EGR valve has some essential system properties such as nonminimum phase behavior in the intake manifold pressure and a non-minimum phase behavior and a sign re- 


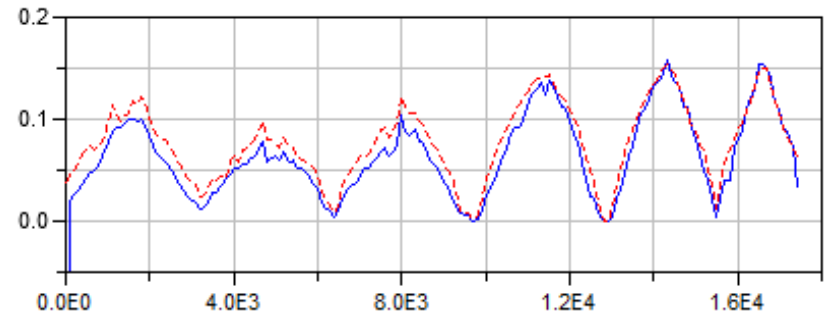

Figure 15: EGR flow model validation. EGR flow $[\mathrm{kg} / \mathrm{s}]$, simulated (solid) and measured (dashed)

versal in the compressor flow [15]. Fig. 16 shows that the the model captures the non-minimum phase behavior between the EGR valve position, $u_{e g r}$, change and inlet manifold pressure, $p_{i n}$.
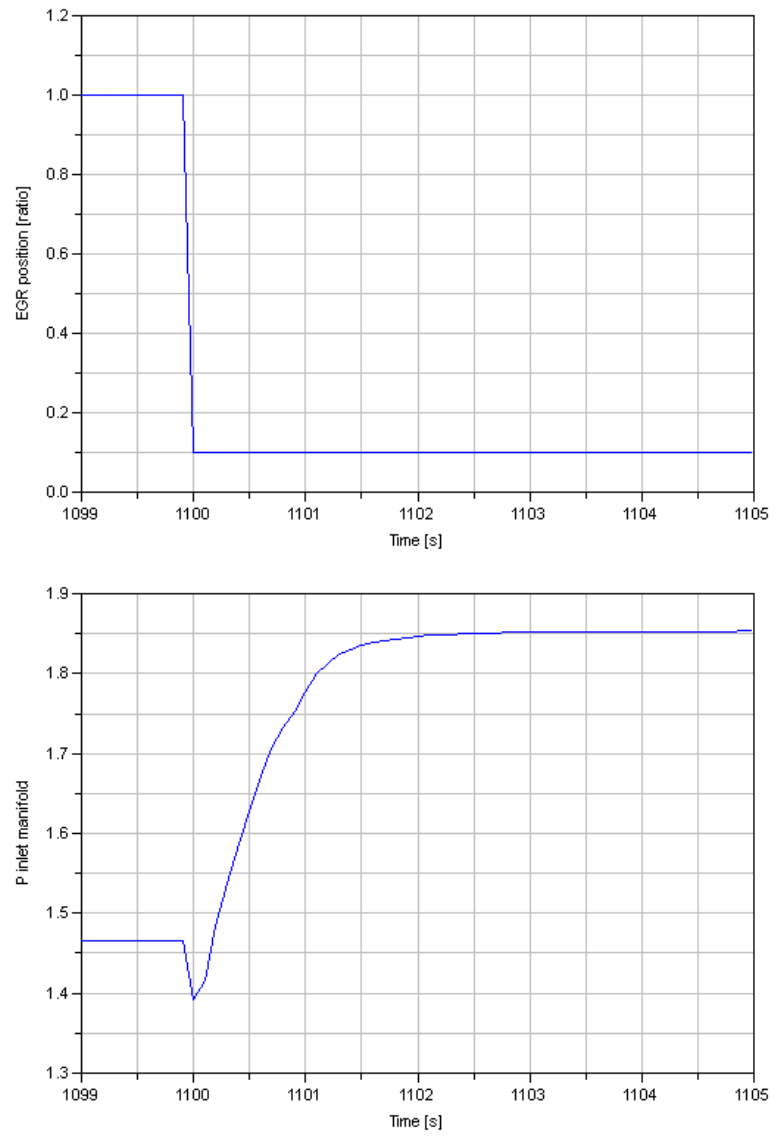

Figure 16: Dynamic verification of the non-minimum phase between $u_{\text {egr }}$ and $p_{\text {in }}$ using steps. Operating point: $\omega_{e}=1500 \mathrm{rpm}, T_{e}=670 \mathrm{Nm}, u_{v g t}=0.5 \mathrm{ra}-$ tio.

Fig. 17 shows that the model capture the nonminimum phase behavior between the VGT position, $u_{v g t}$, and the compressor mass flow $\dot{m}_{c}$. Notice that initially the DC gain between $u_{v g t}$ and $\dot{m}_{c}$ is negative but after a while it becomes positive. This phenomena is even better seen in Fig. 18 where the $u_{v g t}$ is slowly changed from complete opened vanes towards closed position. As the sweep is performed slowly and the other operating conditions are kept constant, the results can be regarded as steady state results.
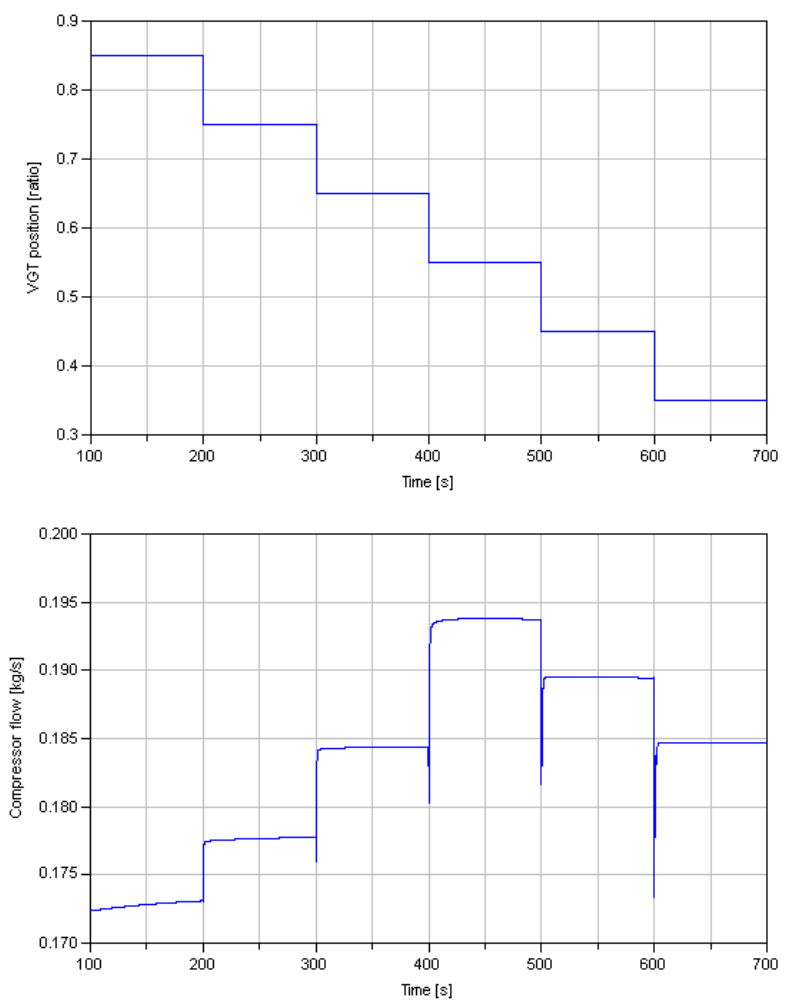

Figure 17: Dynamic verification of the non-minimum phase between $u_{v g t}$ and $\dot{m}_{c}$ using steps. Operating point: $\omega_{e}=1500 \mathrm{rpm}, T_{e}=670 \mathrm{Nm}, u_{e g r}=1$ ratio.
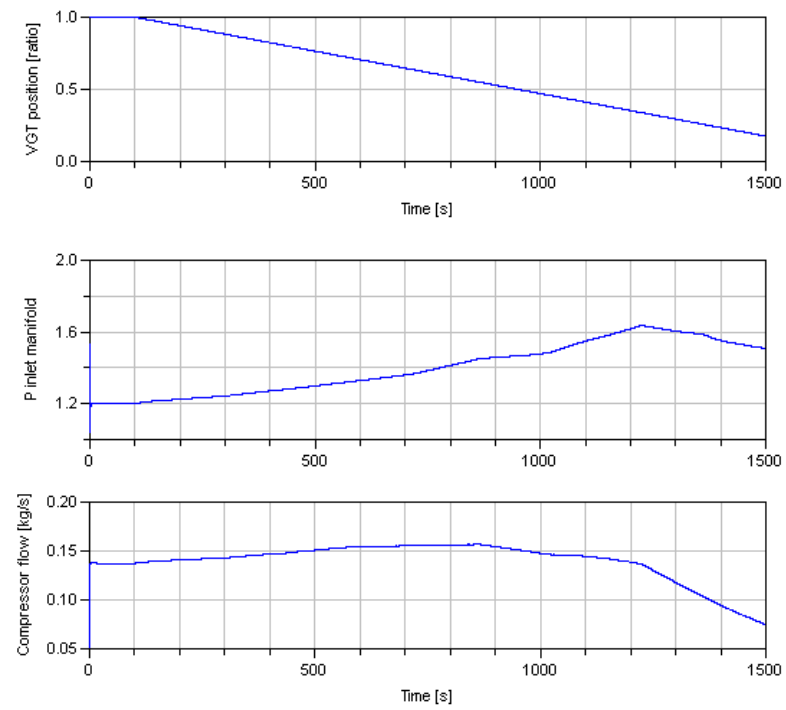

Figure 18: Slow sweep of the $u_{v g t}$ from fully open towards closed position. Operating point: $\omega_{e}=1500$ $\mathrm{rpm}, T_{e}=670 \mathrm{Nm}, u_{e g r}=1$ ratio. 


\subsection{Dynamic validation}

The engine system model is validated with JE05 boundary conditions using the experiment setup in Fig. 9. The cycle is 1830 seconds long and the simulation time for the whole cycle was 735 seconds $(2.5 \mathrm{x}$ faster than real-time) on a standard laptop. The JE05 is a very transient cycle which contains mostly city driving with some high way driving. The engine speed variations during the complete cycle are shown in Fig. 19 and the load variations are shown in Fig. 20.

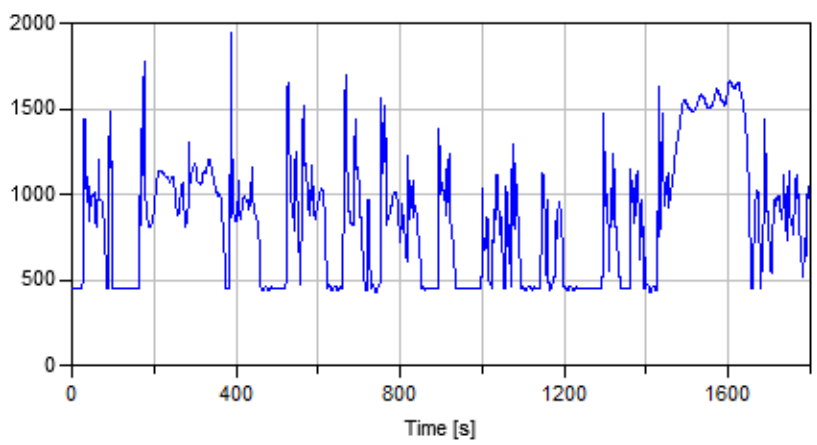

Figure 19: JE05 engine speed [rpm]

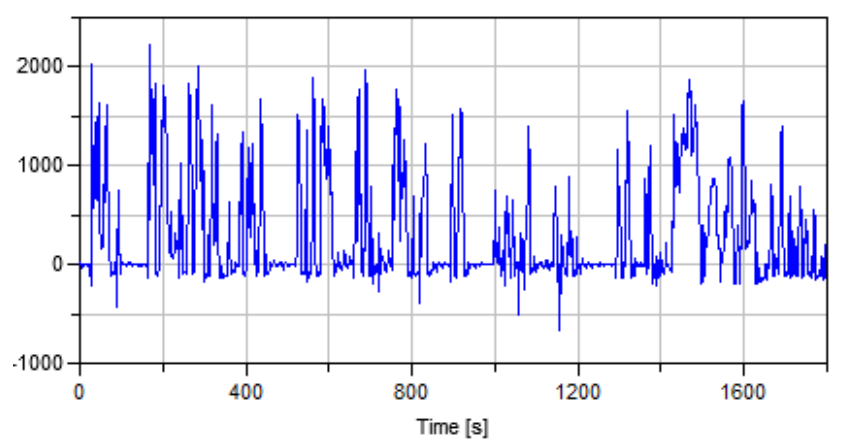

Figure 20: JE05 engine torque [Nm]

The resulting full cycle exhaust gas temperature is shown in Fig. 21 and NOx emissions are shown in Fig. 22. Both the modeled exhaust temperature and the NOx emission captures most of the behavior. The modeled exhaust temperature differs from the measured temperature in the end of the JE05 cycle. The temperature before the VGT capture the temperature behavior correct also in the end of the cycle this indicate that there are still heat transfer effects that need to be incorporated in the model.

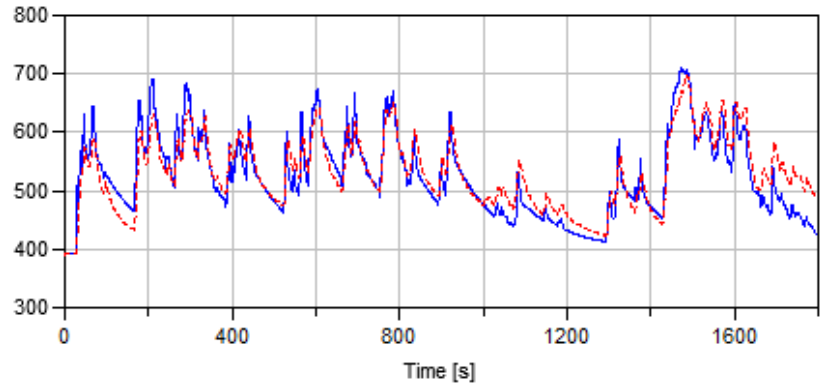

Figure 21: Complete model validation. Exhaust gas temperature $[\mathrm{K}]$, simulated (solid) and measured (dashed)

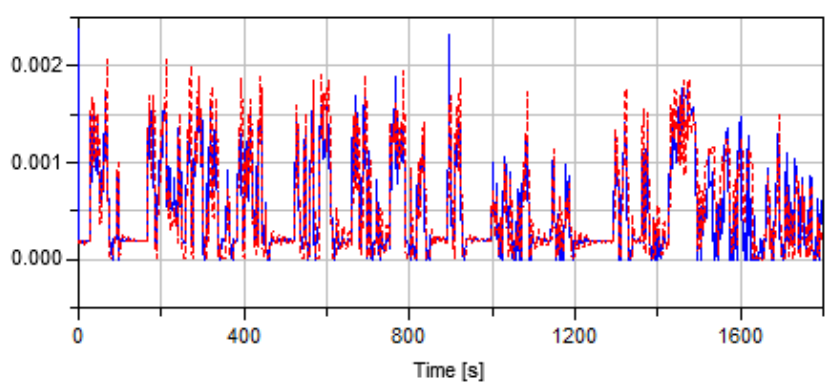

Figure 22: Complete model validation. Exhaust NOx concentration $[\mathrm{kg} / \mathrm{kg}]$, simulated (solid) and measured (dashed)

Figures 23 - 27 show simulation results for engine torque, mass flow rates and exhaust gas temperature from a part of the cycle $(750-1000 \mathrm{~s})$. The exhaust gas temperature is measured in the pipe 1 meter after the turbine. As can be seen in Fig. 23 the model captures most of the behavior. Figures $24-26$ show that the model captures the dynamics of the exhaust, EGR and air mass flows. The ERG flow in Fig. 25 shows a small time lag of the measured flow compared to the simulated. This is likely due to a time lag in the EGR flow sensor.

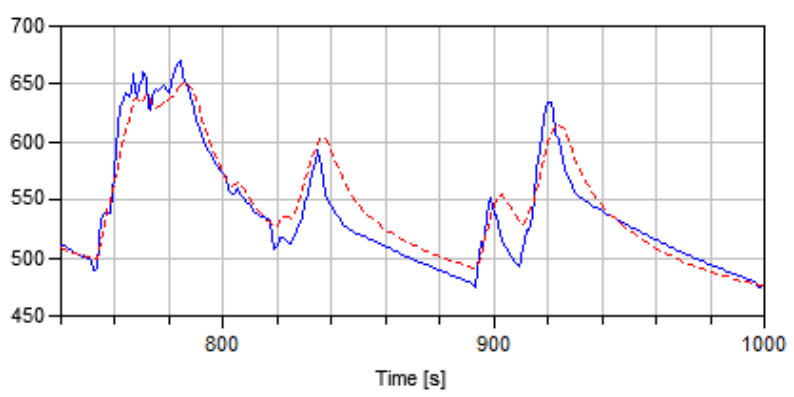

Figure 23: Complete model validation. Exhaust gas temperature $[\mathrm{K}]$, simulated (solid) and measured (dashed) 


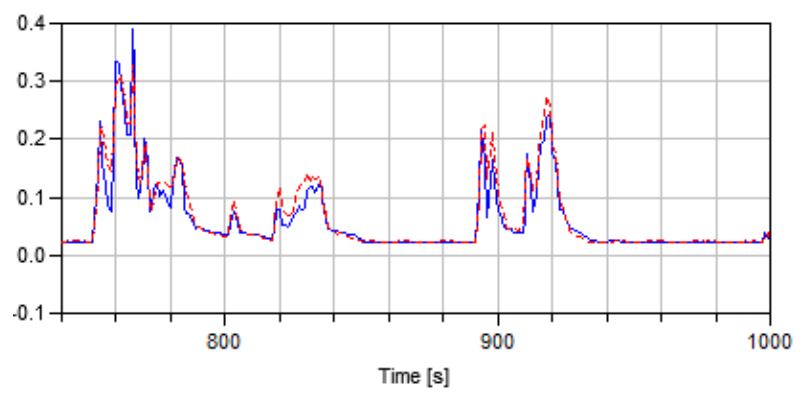

Figure 24: Complete model validation. Exhaust gas flow rate $[\mathrm{kg} / \mathrm{s}]$, simulated (solid) and measured (dashed)

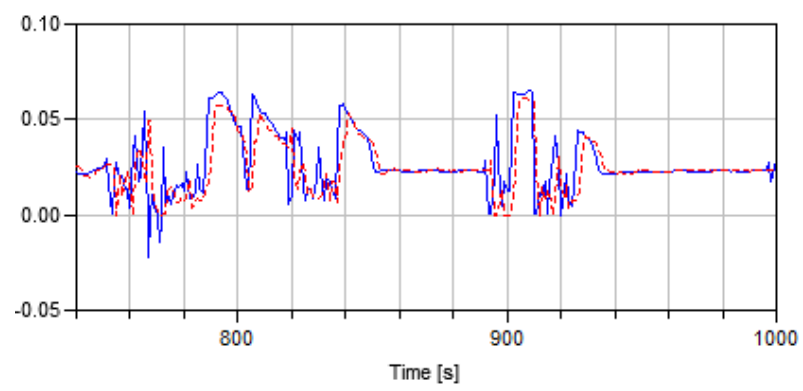

Figure 25: Complete model validation. EGR flow rate $[\mathrm{kg} / \mathrm{s}]$, simulated (solid) and measured (dashed)

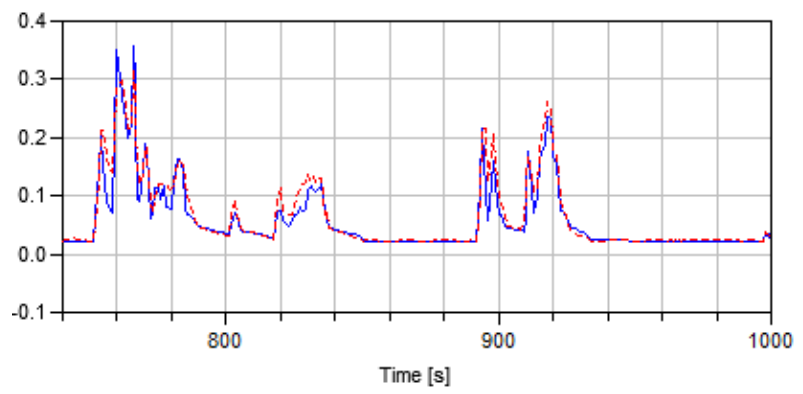

Figure 26: Complete model validation. Air flow rate $[\mathrm{kg} / \mathrm{s}]$, simulated (solid) and measured (dashed)

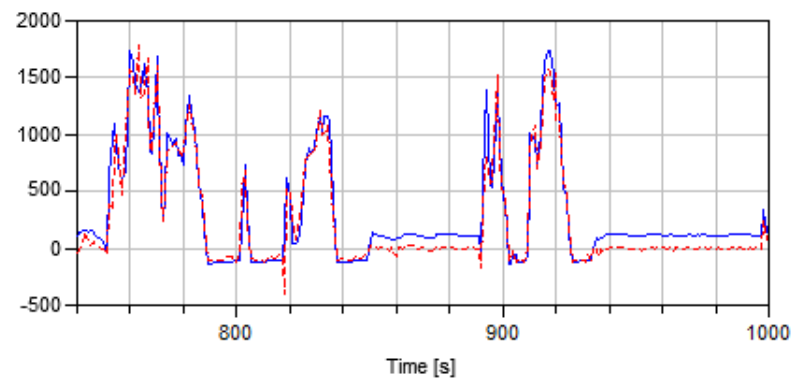

Figure 27: Complete model validation. Engine torque $[\mathrm{Nm}]$, simulated (solid) and measured (dashed)

The model captures most of the dynamics of the engine torque, but for the idling part (e.g. 850-890s) there is an offset between modeled and measured torque (Fig. 27). The difference may be explained by the fact that the friction or the pumping loss measurements which the model is based on are not correct in this region.

Fig. 28 shows the NOx emissions. The black-box model succeeds to capture the behavior. The NOx levels are quite close to the measured level in steady state operation, and the peaks are often quiet close to the measured level regarding timing and level. The NOx level was measured by a Horiba system, which isn't capable of measuring fast transients and the measurements can be regarded as a filtered values.

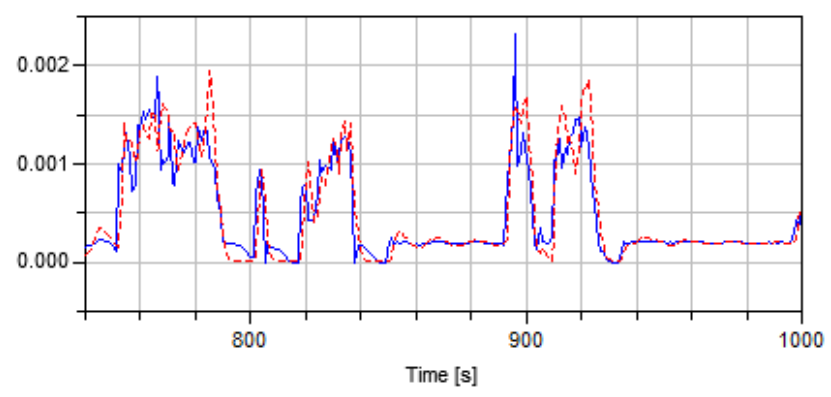

Figure 28: Complete model validation. Exhaust NOx concentration $[\mathrm{kg} / \mathrm{kg}]$, simulated (solid) and measured (dashed)

\section{Discussion}

The components in the 13L Volvo PNLT engine are primarily modeled by a physical first-principle approach. The selected inputs for the emission model does not capture the effect of the wall temperature and a next step can be to parametrize a cylinder wall temperature model in order to model the effects of cold starts. The current simple emission model captures most of the transient effects and in order to further improve the transient optimization based on the models the accuracy needs to be improved. Instead of assuming $\mathrm{CO}_{2}$ in the exhaust manifold based on stoichiometric combustion, it can be added as an output of the emission model. This may improve the estimation of the $\mathrm{CO}_{2}$ in the inlet manifold which is one of the inputs to the emission model. There exists several data driven emission models with similar computational complexity that would be interesting to compare against [16]. The plan for the future is that EDL will be expanded with more combustions model options, including cycle-resolved in-cylinder behavior. By introducing the effects of pressure pulses and improving the internal loss model, the turbo model can also be further improved. 
JModelica.org was used for the optimization of parameters for the heat transfer and thermal dynamics and Dymola was used to export the FMU model. JModelica has extended the Modelica language for increased optimization functionality. The derivative-free simplex method used worked very well for parameter optimization for a model of this complexity without requiring any model modifications. Other tools also exists that can perform calibration using similar methods, for example the model calibration feature in Dymola or Isight. Isight was also tested for the same optimization task and the simplex method available there gave equivalent results to JModelica regarding optimization time and result.

The simulation speed is about 2.5 times faster than real-time using the Dymola integrated Radau solver. This is a variable step-length solver, and the fast average simulation speed does not guarantee that the current model can be used in applications with hard realtime requirements, but this was not in the scope for this model. For hard real-time simulations, fixed-step solvers must be used. This introduces harder requirements on the model regarding fast dynamics and function evaluation time.

As the models of the PNLT engine managed to capture the engine out conditions and the dynamical behavior in the air gas path, the model can be used to develop engine control strategies that reduce the requirement on the EATS. With transient control strategies that reduce transient emissions, the EATS volumes (e.g. DOC, DPF and SCR) may be reduced. Also by adapting the engine control strategies based on the condition of the EATS (e.g. temperature, aging and poisoning) the EATS volumes may be reduced. The fuel cost of the different engine control actions depends significantly on the engine hardware and each has an optimal trade-off between fuel cost and product cost. Engine models based on EDL together with a SIL environment which includes the control strategies is a powerful approach in the investigation of finding the optimal trade-off.

\section{Conclusions}

In this article it has been demonstrated that the newly developed Engine Dynamics library and Dymola can be used for simulation of the gas exchange, transient flow and temperatures and emission trends for a $13 \mathrm{~L}$ Volvo PNLT engine. All components and parameters have been calibrated component wise without any global compensation. Calibration data comes from an engine screening where measurements are made to isolate the different components. Therefore a component can be replaced without any need of a new complete engine screening, allowing for virtual prototyping of new concepts. This is an important advantage compared to black-box modeling of the complete engine, which would require a complete new screening when changing a single component. Finding parameter values for the heat transfer and thermal dynamic in the exhaust that matches measurements is an optimization problem that has been solved using JModelica. The parameters were successfully optimized resulting in good estimation of the exhaust temperature dynamics. The models captured the essential system properties in the gas exchange such as non-minimum phase behavior and sign reversal. As the exhaust mass flow, exhaust temperature and emissions were shown to be well captured the model can be used in order to evaluate control strategies of the air gas management and to find a trade-off between fuel-economy, transient response, engine emissions and EATS requirements. The system identification of the NOx emissions gave good results in the operating area of the JE05 cycle and captured the trends. This indicates that the selected inputs to the emission model contain most of the entities that affect the emissions. Using variable step-length solvers, the engine model simulates faster than realtime for the JE05 cycle. This is a very transient cycle, and therefore the expectation is that other transient cycles will also simulate with real-time like simulation times.

\section{References}

[1] T. Johnson Diesel Emissions in Review, SAE Technical Paper 2011-01-0304, 2011.

[2] R. Cloudt and F. Willems. Integrated Emission Management strategy for cost-optimal engineaftertreatment operation, SAE Technical Paper 2011-01-1310, 2011.

[3] Dymola User Manual, Volume 1, Lund, 2011

[4] J. Batteh, M. Tiller and C. Newman. Simulation of Engine Systems in Modelica, Proceedings of the 3rd Modelica Conference, Linköping, Sweden, 2003.

[5] A. Picarelli and M. Dempsey. Investigating the Multibody Dynamics of the Complete Powertrain System, Proceedings of the 7th Modelica Conference, Como, Italy, 2009. 
[6] L. Guzzella and C.H. Onder. Introduction to Modeling and Control of Internal Combustion Engine Systems, 2nd edition, 2010. ISBN 9783-642-10774-0.

[7] R. Johansson. System modeling \& Identification, 2009. ISBN 0-13-482308-7.

[8] HIH Saravanamuttoo, GFC Rogers and H Cohen. Gas Turbine Theory, Fifth Edition, 2001. ISBN 978-0-13-015847-5.

[9] B.J. McBride, M.J. Zehe and S. Gordon. NASA Glenn Coefficients for Calculating Thermodynamic Properties of Individual Species. NASA report TP-2002-211556, 2002.

[10] J. D'Errico. Understanding GRIDFIT, 2006. Available for download at http://www.mathworks.com/matlabcentral/ fileexchange/8998 (last accessed 20120228).

[11] J. Åkesson, K-E. Årzén, M. Gäfvert, T. Bergdahl and H. Tummescheit. Modeling and Optimization with Optimica and JModelica.org - Language and Tools for Solving Large-Scale Dynamic Optimization Problems, Computers and Chemical Engineering, 34:11, pp. 1737-1749, November 2010

[12] S. Gedda. Calibration of Modelica models using derivative-free optimization, Master's thesis 2011:E46, Lund University, Faculty of Engineering, Centre For Mathematical Sciences, Mathematics, 2011.

[13] S. Gedda, C. Andersson, J. Åkesson and S. Diehl. Derivative-free Parameter Optimization of Functional Mock-up Units. In 9th International Modelica Conference, 2012.

[14] MODELISAR(07006). Functional Mock-up Interface for Model Exchange Available for download at: http://www.functional-mockupinterface.org/ (last accessed 20120228).

[15] J. Wahlström and L. Eriksson. Modeling of a diesel engine with VGT and EGR capturing Sign Reversal and Non-minimum Phase Behavior. Proceedings of the Institution of Mechanical Engineers, Part D, J. of Automobile Engineering, Volume 225, Issue 7, July 2011.

[16] M. Grahn and T. McKelvey. MA Structure and Calibration Method for Data-driven Modeling of
NOX and Soot Emissions from a Diesel Engine. SAE Technical Paper 2012-XX-0351, 2012. 
